\title{
Cambios cefalométricos producidos por Pistas Planas y Bionator en la clase II división 1
}

Cephalometric changes produced by Planas and Bionator tracks in class I/ division 1

\author{
Mudanças cefalométricas produzidas \\ por Pistas Planas e Bionator na classe II divisão 1 \\ Clotilde de la C. Mora Pérez ${ }^{1}$ \\ Ivette Alvarez Mora ${ }^{2}$ \\ Randy Liburd ${ }^{3}$ \\ Ana María Armas Sánchez ${ }^{4}$
}

Recibido: 12 de diciembre de 2017

Aprobado: 16 de agosto de 2018

Publicado: 20 de marzo de 2019

Cómo citar este artículo: Mora-Pérez CDLC, Alvarez-Mora I, Liburd R, Armas-Sánchez AM. Cambios cefalométricos producidos por Pistas Planas y Bionator en la clase II división 1. Revista Nac Odontol. 2019;15(28):1-19 doi: https://doi.org/10.16925/2357-4607.2019.01.02

\footnotetext{
Artículo de investigación. https://doi.org/10.16925/2357-4607.2019.01.02

1 Universidad de Ciencias Médicas de Cienfuegos, Cuba https://orcid.org/0000-0002-9862-7199 Correo electrónico: clotirdecmp@jagua.cfg.sld.cu

2 Clínica Estomatológica Especialidades, Cienfuegos, Cuba https://orcid.org/0000-0002-2671-6360

3 Clínica Sant Kist y Nevis https://orcid.org/0000-0001-9287-0609

4 Clínica Raúl González, Cienfuegos, Cuba https://orcid.org/0000-0002-9022-6745
} 


\section{Resumen}

Introducción: el Bionator y las pistas planas son aparatos funcionales que se encuentran entre las opciones de tratamiento de la maloclusión clase Il división 1.

Objetivo: comparar los cambios cráneofaciales y de tejidos blandos resultantes del tratamiento ortopédico para la corrección de maloclusión clase II división 1.

Métodos: se realizó un estudio de intervención, cuasiexperimental, la unidad muestral fue de 622 niños entre 6 y 9 años de ambos sexos de la Escuela Primaria Guerrillero Heroico del área II del municipio Cienfuegos, la muestra quedó constituida por 20 pacientes, se dividió en 2 grupos equitativamente y se utilizaron como terapéutica los aparatos funcionales de Pistas Planas y el Bionator. Se tomaron telerradiografías laterales de cráneo al inicio y al año de tratamiento, se realizaron mediciones lineales y angulares de Steiner, Ricketts y McNamara. Se evaluaron los cambios cefalométricos esqueletales y de tejidos blandos antes y después del tratamiento.

Resultados: se observaron cambios en las mediciones de las radiografías laterales de cráneo, con variaciones cefalométricas cráneofaciales de significación estadística importante. En los tejidos blandos aumentó el ángulo nasolabial, el cual se encontraba disminuido debido al componente labial ya que existía protrusión ligera del labio superior.

Conclusión: se comprobó desde el punto de vista estadístico que los pacientes con pistas planas presentaron cambios craneofaciales estadísticamente más significativos en la mayoría de las medidas cefalométricas, los cambios en los tejidos blandos no fueron significativos para ninguno de las dos terapéuticas empleadas y el biotipo fue modificado positivamente con ambas aparatologías

Palabras clave: maloclusión, aparatos activadores, aparatos ortodóncicos funcionales, Bionator, Pistas Planas.

\section{Summary}

Introduction: The Bionator and flat tracks are functional appliances that are among the treatment options for class II malocclusion division 1.

Objective: To compare the craniofacial and soft tissue changes resulting from orthopedic treatment to correct the class II division 1 malocclusion.

Methods: an almost experimental intervention study was carried out; the sample unit was 622 children between 6 and 9 years of age, of both sexes, of the Guerrillero Heroico Primary School, area II of the Cienfuegos municipality; Of these, 20 patients were selected, divided into two groups equally, with which the functional appliances of Pistas Planas and Bionator were used as therapy. Lateral cephalograms of the skull were taken at the beginning and after one year of treatment; linear and angular measurements were performed by Steiner, Ricketts and McNamara. Cephalometric changes in skeletal and soft tissues were evaluated before and after treatment.

Results: Changes were observed in measurements of lateral cranial radiographs, with cephalometric craniofacial variations of significant statistical significance. In the soft tissues, the nasolabial angle increased, which was diminished due to the labial component, since there was light protrusion of the upper lip.

Conclusion: it was statistically proven that patients with flat tracks had statistically more significant craniofacial changes in most cephalometric measurements; soft tissue changes were not significant for any of the two therapies employed, and the biotype was positively modified with both devices.

Keywords: malocclusion, activating appliances, functional orthodontic appliances, Bionator, Flat Tracks. 


\section{Resumo}

Introdução: o Bionator e as pistas planas são aparelhos funcionais que se encontram entre as opções de tratamento da má-oclusão classe II divisão 1.

Objetivo: comparar as mudanças craniofaciais e de tecidos moles resultantes do tratamento ortopédico para corrigir a má-colusão classe II divisão 1.

Métodos: foi realizado um estudo de intervenção, quase experimental; a unidade de amostra foi de 622 crianças entre 6 e 9 anos, de ambos os sexos, da Escuela Primaria Guerrillero Heroico, da área II do município Cienfuegos; destas, foram selecionados 20 pacientes, divididos em dois grupos equitativamente, com os quais foram utilizados, como terapia, os aparelhos funcionais de Pistas Planas e Bionator. Foram tomadas telerradiografias laterais de crânio no início e depois de um ano do tratamento; foram realizadas medições lineares e angulares de Steiner, Ricketts e McNamara. Foram avaliadas as mudanças cefalométricas esqueletais e tecidos moles antes e depois do tratamento.

Resultados: foram observadas mudanças nas medições das radiografias laterais de crânio, com variações cefalométricas craniofaciais de significação estatística importante. Nos tecidos moles, aumentou o ângulo nasolabial, o qual se encontrava diminuído devido ao componente labial, já que existia protrusão leve do lábio superior.

Conclusão: foi comprovado, do ponto de vista estatístico, que os pacientes com pistas planas apresentaram mudanças craniofaciais estatisticamente mais significativas na maioria das medidas cefalométricas; as mudanças nos tecidos moles não foram significativas para nenhuma das duas terapias empregadas, e o biotipo foi modificado positivamente com ambas as aparatologias.

Palavras-chave: má-oclusão, aparelhos ativadores, aparelhos ortodônticos funcionais, Bionator, Pistas Planas.

\section{Introducción}

La sonrisa es la puerta de entrada a las relaciones humanas, por esta razón se le da mucha importancia al cuidado de los dientes en especial a su alineación en el arco dentario. La oclusión normal es aquel contacto en ausencia de patologías del aparato masticatorio, que tiene la capacidad de adaptarse a pequeñas desviaciones dentro de un límite de tolerancia (1).

Sin embargo, la maloclusión es una condición donde su presencia causa problemas funcionales al sistema cráneo-mandibular, así como un impacto negativo sobre la calidad de vida de los niños como de sus familias (1, 2).

La clasificación más utilizada universalmente es la que presentó Edward $\mathrm{H}$. Angle, basado en las relaciones mesiodistales de los primeros molares permanentes y describió las diferentes maloclusiones denominadas CLASES (1, 3, 4).

La prevalencia de maloclusiones indica que aproximadamente un tercio de la población tiene una oclusión que puede considerarse como normal o casi normal, mientras que unos dos tercios tienen algún grado de maloclusión. La clase II División 1 es la desarmonía dentoesquelética más frecuente en la población de raza blanca que -en mayor porcentaje- llega a la consulta en búsqueda de tratamiento (5). 
Se plantea que su etiopatogenia suele estar relacionada con factores extrínsecos como pueden ser los hábitos, ellos constituyen un freno patológico para el desarrollo de la arcada mandibular y un estímulo para el prognatismo maxilar (1). La maloclusión clase II se caracteriza por presentar un retrognatismo mandibular o prognatismo maxilar, que afecta la relación molar $(6,7)$.

El síndrome de clase II es una de las desarmonías esqueletales que pueden aparecer a nivel del macizo cráneo-facial en el niño durante el crecimiento y desarrollo, éste suele ocasionar impactos negativos en la esfera emocional debido a la afectación estética a la que conlleva. Se debe aclarar que esta implicación estética se debe a una discrepancia de tamaño entre el maxilar y la mandíbula, por estas razones justifican el tratamiento temprano de dichas alteraciones, siendo el avance mandibular una de las alternativas para su corrección (8-12).

La Aparatología de Ortopedia Funcional (AOF) promueve la corrección de los desequilibrios funcionales, esqueléticos y dentales, y recupera los estímulos de crecimiento adecuados que se han perdido (13).

Una estrategia común en su tratamiento es corregir u ocultar la discrepancia esqueletal, las terapéuticas más efectivas son los aparatos funcionales, en una fase temprana para optimizar el desarrollo del esqueleto facial, ya que su acción principal es sobre los músculos y los huesos, secundariamente sobre las piezas dentarias (14-16).

Uno de los "aparatos funcionales" mono-maxilares más populares utilizados en el presente es el Bionator, debido a su simplicidad, fuerza, a su fácil construcción, a la aceptación del paciente y su efectividad cuando se utiliza durante el periodo de máximo pico - surto- de crecimiento $(16,17)$. Tiene el objetivo de establecer una buena coordinación de los músculos y eliminar las posibles restricciones deformadoras del crecimiento, al mismo tiempo que elimina las cargas ejercidas sobre el cóndilo mediante una posición adelantada de la mandíbula (16).

Las Pistas Planas es otro aparato funcional de acción bimaxilar indicado para la corrección de la clase II esqueletica, basados en la aplicación de la terapéutica de Rehabilitación Neuroclusal creada por el Doctor Pedro Planas. Su principio biológico es establecer un plano oclusal fisiológico con libertad de los movimientos mandibulares y así se corrigen las relaciones maxilo-mandibulares $(15,16)$.

Para esta investigación se trazó como objetivo comparar los cambios cráneofaciales y de tejidos blandos resultantes del tratamiento ortopédico con el uso del Bionator y las Pistas Planas para la corrección de maloclusión clase II división 1, en niños entre 6 y 9 años de ambos sexos de la Escuela Primaria Guerrillero Heroico del área II del municipio Cienfuegos. Evaluar los cambios cefalométricos cráneofaciales 
producidos por el uso del estos aparatos durante el tiempo de tratamiento, así como comparar los cambios cefalométricos en el perfil blando producidos en los pacientes objeto de estudio.

\section{Materiales y métodos}

Se realizó un estudio de intervención cuasiexperimental para evaluar los cambios cefalométricos esqueletales y de tejidos blandos, producidos por el uso de las Pistas Planas y el Bionator en pacientes con maloclusión de clase II división I de Angle, al año de tratamiento. El trabajo se realizó en el período comprendido de octubre de 2015 a octubre de 2016.

\section{Universo y muestra de estudio}

La unidad muestral fue de 622 niños entre seis y nueve años de ambos sexos de la Escuela Primaria Guerrillero Heroico del área II del municipio Cienfuegos. Se utilizó una muestra no probabilística a conveniencia por cumplir los criterios de inclusión, así quedó constituida por 20 pacientes, la cual se dividió en 2 grupos equitativamente, buscando un equilibrio entre las edades y el sexo presentes en ambos y no existiendo diferencias significativas entre éstos, se utilizó como terapéutica los aparatos funcionales de Pistas Planas y el Bionator.

Se consideraron como criterios de inclusión: edad entre 6 y 9 años, resalte incisivo mayor de $7 \mathrm{~mm}$, sobrepase incisivo mínimo de 2/3 de corona, relación molar de distoclusión en ambos lados excluyendo relación de cúspide-cúspide por estar presente los segundos molares temporales, retrognatismo mandibular predominante sobre la protrusión maxilar, correcta relación transversal inter-arcada, inicial y durante las maniobras de avance mandibular, sin tratamiento ortodóncico previo y contar con el consentimiento de padres/tutores a participar en la investigación.

Se consideró como criterio de exclusión la existencia de relación molar de distoclusión bilateral con relación molar de cúspide-cúspide donde estén presentes los segundos molares temporales inferiores por el fenómeno del corrimiento mesial tardío.

\section{Consideraciones éticas}

Esta investigación fue aprobada por un comité de ética acreditado, además se obtuvo la autorización de la Dirección Provincial de Educación, también se obtuvo el consentimiento informado de los padres de los escolares y tutores, participantes en el estudio. 
6 Cambios cefalométricos producidos por Pistas Planas y Bionator en la clase II división 1

\section{Método}

Los grupos así conformados usaron las Pistas Planas y el Bionator construidos según diseños y teniendo en cuenta las magnitudes de la mordida constructiva, en que se siguieron las normas generales definidas en la bibliografía consultada.

Los aparatos se confeccionaron en el laboratorio siguiendo los requisitos descritos por el Doctor Pedro Planas y el Doctor Wilhelm Balters para la elaboración de las Pistas de Rodaje Horizontal para clase II y el Bionator del tipo clase II respectivamente.

Los aparatos funcionales se confeccionaron siguiendo los criterios descritos por el Doctor Wilhelm Balters y el Doctor Pedro Planas y para la elaboración del Bionator $^{18}$ del tipo clase II y las Pistas (19) para clase II respectivamente con una mordida de construcción de parafina en un articulador, de acuerdo con la posición de oclusión de resalte funcional de los dientes anteriores. Una vez confeccionados e instalados los aparatos funcionales, el primer control se realizó a la semana, para detectar molestias y verificar la adaptación. Luego fueron citados los pacientes cada cuatro semanas para realizar los ajustes necesarios, se mantuvieron con el aparato un año; lo usaron todo el tiempo excepto para comer.

Tanto para el diagnóstico como para evaluar los cambios al año de tratamiento, se tomaron telerradiografías laterales de cráneo, la cuales fueron medidas por uno de los autores del presente estudio, aplicando mediciones lineales o angulares de Steiner, Ricketts y McNamara. Además de las variables edad - seis, siete, ocho y nueve años-y sexo -masculino, femenino-, se analizaron, mediante diferentes mediciones cefalométricas. Los siguientes son los parámetros, antes de usar el aparato y al año de usarlo:

- Patrón de crecimiento según (ángulo Y-SN).

- Patrón esqueletal según Steiner (ángulos SNA, SNB y ANB).

- Patrón esqueletal según McNamara (distancia entre el punto A y la perpendicular $\mathrm{Na}$, distancia desde el pogonion hasta la perpendicular $\mathrm{Na}$, longitud efectiva del maxilar, longitud efectiva de la mandíbula y altura anteroinferior de la cara).

- Patrón esqueletal según Ricketts (convexidad facial, ángulo del eje facial, ángulo de la profundidad facial, ángulo del plano mandibular, ángulo de la altura facial inferior y ángulo del arco mandibular).

- Perfil blando (ángulo nasolabial (Legan Burstone) y protrusión labial (Ricketts)). Para visualizar el perfil facial, las radiografías fueron analizadas con el método de reducción de radiaciones del área de tejidos blandos. En los calcos cefalométricos se realizaron mediciones angulares y lineales de tejidos duros y de tejidos blandos, según las mediciones cefalométricas de Ricketts. 
- Biotipo facial: dolicofacial, dólico suave y mesofacial (Rickett). Además, se calculó teniendo en cuenta las cinco medidas de Ricketts: eje facial, profundidad facial, ángulo del plano mandibular, altura facial inferior y arco mandibular (20).

Los datos fueron registrados en un formulario confeccionado al efecto. Para procesar la información se utilizó el paquete estadístico sPSS V.15.0. (Statistical Package for the Social Sciences). Se realizó un análisis descriptivo de la información recopilada. El análisis estadístico se realizó mediante tablas de distribución de frecuencia, estadísticos de tendencia central y de dispersión, pruebas de hipótesis paramétricas y no paramétricas para comparar medias, como correlaciones de Pearson. Se compararon los valores de las variables analizadas (medidas cefalométricas) mediante la Prueba T, que permite comparar muestras independientes, para la cual se prefijó un nivel de significación de $5 \%$.

\section{Resultados}

La tabla 1 muestra el comportamiento demográfico de la población en estudio, donde existió un predominio del sexo masculino, principalmente en la edad de 8 años con un $66,7 \%$, mientras que en el sexo femenino fue a los 7 años con $57,1 \%$.

Tabla 1. Distribución demográfica de la población objeto de estudio según edad y sexo.

\begin{tabular}{|c|c|c|c|c|c|c|c|c|c|c|}
\hline \multirow{3}{*}{ SEXO } & \multicolumn{8}{|c|}{ EDAD } & \multirow{2}{*}{\multicolumn{2}{|c|}{ TOTAL }} \\
\hline & \multicolumn{2}{|c|}{6 Años } & \multicolumn{2}{|c|}{7 Años } & \multicolumn{2}{|c|}{8 Años } & \multicolumn{2}{|c|}{9 Años } & & \\
\hline & No & $\%$ & No & $\%$ & No & $\%$ & No & $\%$ & No & $\%$ \\
\hline Masculino & 1 & 50,0 & 3 & 42,9 & 6 & 66,7 & 1 & 50,0 & 11 & 55,0 \\
\hline Femenino & 1 & 50,0 & 4 & 57,1 & 3 & 33,3 & 1 & 50,0 & 9 & 45,0 \\
\hline Total & 2 & 100,0 & 7 & 100,0 & 9 & 100,0 & 2 & 100,0 & 20 & 100,0 \\
\hline
\end{tabular}

Fuente: elaboración propia

En la tabla 2 se analizó el comportamiento en el patrón de crecimiento, predominó la tendencia al crecimiento horizontal antes y después de aplicar la terapéutica funcional. Para las Pistas Planas se encontró un promedio inicial del ángulo $Y$ - SN de 60,50 que se elevó a $65,60^{\circ}$ y los del Bionator, $64,50^{\circ}$ inicialmente y $65,50^{\circ}$ al año de tratamiento. Existieron diferencias estadísticas significativas para la terapia con Pistas Planas 
Tabla 2. Comportamiento en el patrón de crecimiento con la terapéutica funcional de las Pistas Planas y el Bionator.

\begin{tabular}{|c|c|c|c|c|c|}
\hline \multicolumn{3}{|c|}{ Pistas Planas } & \multicolumn{3}{|r|}{ Bionator } \\
\hline \multicolumn{2}{|c|}{ Valor promedio } & \multirow{2}{*}{ Significación estadística } & \multicolumn{2}{|c|}{ Valor promedio } & \multirow{2}{*}{ Significación estadística } \\
\hline Antes & Después & & Antes & Después & \\
\hline $60,50^{\circ}$ & $65,60^{\circ}$ & 0,015 & $64,50^{\circ}$ & $65,60^{\circ}$ & 0,221 \\
\hline
\end{tabular}

Fuente: elaboración propia

En la evaluación cefalométrica del patrón esqueletal, antes y después del uso de ambos aparatos, se pudo constatar que, después del tratamiento, aumentó el ángulo SNB, predominó el incremento con el uso de las Pistas Planas, el cual varió de $77,0^{\circ}$ a $79,7^{\circ}$. El ángulo ANB en clase II, disminuyó con ambos aparatos significativamente.

Tabla 3. Evaluación cefalométrica del patrón esqueletal según el análisis de Steiner.

\begin{tabular}{|c|c|c|c|c|c|c|}
\hline \multirow{3}{*}{$\begin{array}{c}\text { Medidas } \\
\text { cefalométricas }\end{array}$} & \multicolumn{3}{|c|}{ Pistas Planas } & \multicolumn{3}{|c|}{ Bionator } \\
\hline & \multicolumn{2}{|c|}{ Valor promedio } & \multirow{2}{*}{$\begin{array}{l}\text { Significación } \\
\text { estadística }\end{array}$} & \multicolumn{2}{|c|}{ Valor promedio } & \multirow{2}{*}{$\begin{array}{l}\text { Significación } \\
\text { estadística }\end{array}$} \\
\hline & Antes & Después & & Antes & Después & \\
\hline SNA & $83,20^{\circ}$ & $83,40^{\circ}$ & 0,168 & $82,20^{\circ}$ & $82,40^{\circ}$ & 0,168 \\
\hline SNB & $77,00^{\circ}$ & $79,70^{\circ}$ & 0,00 & $76,90^{\circ}$ & $78,10^{\circ}$ & 0,003 \\
\hline ANB & $6,20^{\circ}$ & $3,70^{\circ}$ & 0,00 & $5,30^{\circ}$ & $4,40^{\circ}$ & 0,019 \\
\hline
\end{tabular}

Fuente: elaboración propia

Se logró variación de la posición anteroposterior del Pogonion con predominio con el uso de las Pistas Planas que, con el Bionator, de $-5,1 \mathrm{~mm}$ a $-2,9 \mathrm{~mm}$ y $-4,7 \mathrm{~mm}$ a $-4,1 \mathrm{~mm}$ respectivamente. Ambas terapias mostraron valores estadísticamente significativos al año de tratamiento.

La longitud maxilar en estos pacientes se modificó muy pobremente pero continúa en la norma. Sin embargo, la longitud mandibular efectiva al año de tratamiento ortopédico se modificó presentando un aumento con ambas terapias, pero particularmente con las Pistas Planas, con diferencias estadísticamente significativas. Sin embargo continúa la hipoplasia mandibular.

La altura facial anteroinferior disminuyó con la utilización de ambos aparatos, principalmente con las Pistas Planas de 63,8 mm a 61,6 mm, con significación estadística para este aparato. 
Tabla 4. Evaluación cefalométrica del patrón esqueletal según el análisis de Mc Namara.

\begin{tabular}{|c|c|c|c|c|c|c|}
\hline \multirow{3}{*}{$\begin{array}{l}\text { Medidas cefalométricas } \\
\qquad(\mathrm{mm})\end{array}$} & \multicolumn{3}{|c|}{ Pistas Planas } & \multicolumn{3}{|c|}{ Bionator } \\
\hline & \multicolumn{2}{|c|}{ Valor promedio } & \multirow{2}{*}{$\begin{array}{l}\text { Significación } \\
\text { estadística }\end{array}$} & \multicolumn{2}{|c|}{ Valor promedio } & \multirow{2}{*}{$\begin{array}{l}\text { Significación } \\
\text { estadística }\end{array}$} \\
\hline & Antes & Después & & Antes & Después & \\
\hline Punto A - Na perpendicular & 2,00 & 1,00 & 0,008 & 1,00 & 0,80 & 0,168 \\
\hline Pogonion - Na perpendicular & $-5,10$ & $-2,90$ & 0,005 & $-4,70$ & $-4,10$ & 0,024 \\
\hline Longitud maxilar Efectiva & 86,00 & 88,10 & 0,001 & 89,40 & 90,00 & 0,005 \\
\hline Longitud mandíbular Efectiva & 103,6 & 106,8 & 0,00 & 105,3 & 106,7 & 0,003 \\
\hline Altura Facial Anteroinferior & 63,80 & 61,60 & 0,002 & 62,40 & 61,50 & 0,054 \\
\hline
\end{tabular}

Fuente: elaboración propia

Al realizar el análisis del patrón esqueletal en la tabla 5, se observó que la convexidad facial disminuyó con ambas terapias, siendo la disminución mayor con las Pistas Planas cuyo valor promedio osciló de 5,3 mm a 3,2 mm y en el Bionator fue de 3,9 $\mathrm{mm}$ a 3,4 mm. Ambos resultados se encuentran en la norma, y muestran valores con significación estadística para las Pistas Planas. Respecto al ángulo del eje facial se elevó, sufriendo modificaciones estadísticamente significativas con las Pistas Planas. Con el uso del Bionator, hubo cambios estadísticamente no significativos y una ligera apertura del eje. En general, en la evaluación cefalométrica del patrón esqueletal según el análisis de Ricketts los valores promedio en ambos grupos antes y después están en la norma.

El comportamiento de la profundidad facial tras un año de tratamiento se elevó en ambos aparatos con valores estadísticamente significativos. Sin embargo, el plano mandibular prácticamente no se modificó durante el tratamiento, mostrando valores de significación no estadística en ninguna de las dos terapias aplicadas.

El ángulo de la altura facial inferior disminuyó mostrando valores con significación estadística solamente con las Pista Planas.

Con la aplicación de ambas terapias el arco mandibular mostró cambios positivos, principalmente en las Pistas Planas de $26,9^{\circ}$ a $27,5^{\circ}$. 
Tabla 5. Evaluación cefalométrica del patrón esqueletal según el análisis de Ricketts.

\begin{tabular}{|c|c|c|c|c|c|c|}
\hline \multirow{3}{*}{$\begin{array}{c}\text { Medidas } \\
\text { cefalométricas }\end{array}$} & \multicolumn{3}{|c|}{ Pistas Planas } & \multicolumn{3}{|c|}{ Bionator } \\
\hline & \multicolumn{2}{|c|}{ Valor promedio } & \multirow{2}{*}{$\begin{array}{l}\text { Significación } \\
\text { estadística }\end{array}$} & \multicolumn{2}{|c|}{ Valor promedio } & \multirow{2}{*}{$\begin{array}{l}\text { Significación } \\
\text { estadística }\end{array}$} \\
\hline & Antes & Después & & Antes & Después & \\
\hline Convexidad Facial(mm) & 5,30 & 3,20 & 0,002 & 3,90 & 3,40 & 0,052 \\
\hline Eje Facial & $86,80^{\circ}$ & $88,40^{\circ}$ & 0,00 & $86,80^{\circ}$ & $86,30^{\circ}$ & 0,614 \\
\hline Profundidad Facial & $84,50^{\circ}$ & $86,50^{\circ}$ & 0,00 & $84,50^{\circ}$ & $85,10^{\circ}$ & 0,005 \\
\hline Plano Mandibular & $27,20^{\circ}$ & $27,00^{\circ}$ & 0,693 & $27,40^{\circ}$ & $27,50^{\circ}$ & 0,678 \\
\hline Altura Facial Inferior & $46,80^{\circ}$ & $45,80^{\circ}$ & 0,002 & $47,30^{\circ}$ & $46,70^{\circ}$ & 0,054 \\
\hline Arco Mandibular & $26,90^{\circ}$ & $27,50^{\circ}$ & 0,024 & $25,90^{\circ}$ & $26,30^{\circ}$ & 0,104 \\
\hline
\end{tabular}

Fuente: elaboración propia

En la tabla 6 se puede observar que el ángulo nasolabial aumentó con ambas terapias sin mostrar significación estadística alguna. Por otra parte, la protrusión labial no sufrió prácticamente modificación alguna con el uso de ambas técnicas. Aumentó discretamente con las Pistas Planas y se comportó de forma inversa en el Bionator, pero no mostró significación estadística alguna, ya que desde el inicio presentaban ligera protusión labial.

Tabla 6. Comparación cefalométrica de los cambios producidos en el perfil blando con el uso del Bionator y las Pistas Planas.

\begin{tabular}{|c|c|c|c|c|c|c|}
\hline \multirow{3}{*}{$\begin{array}{c}\text { Medidas } \\
\text { cefalométricas }\end{array}$} & \multicolumn{3}{|c|}{ Pistas Planas } & \multicolumn{3}{|c|}{ Bionator } \\
\hline & \multicolumn{2}{|c|}{ Valor promedio } & \multirow{2}{*}{$\begin{array}{l}\text { Significación } \\
\text { estadística }\end{array}$} & \multicolumn{2}{|c|}{ Valor promedio } & \multirow{2}{*}{$\begin{array}{l}\text { Significación } \\
\text { estadística }\end{array}$} \\
\hline & Antes & Después & & Antes & Después & \\
\hline Ángulo nasolabial & $101,4^{\circ}$ & $108,1^{\circ}$ & 0,059 & $104,4^{\circ}$ & $106,5^{\circ}$ & 0,189 \\
\hline $\begin{array}{l}\text { Protrusión la- } \\
\text { bial(mm) }\end{array}$ & 0,6 & 0,9 & 0,434 & 3,0 & 2,5 & 0,475 \\
\hline
\end{tabular}

Fuente: elaboración propia

Como se puede observar en la tabla 8 , al inicio de la terapéutica funcional con las Pistas Planas, hubo predominio de los Mesofaciales y con el Bionator fueron los Dólico suave. Tras un año de tratamiento con los aparatos funcionales, se observó que la mitad de los pacientes Dólico suave tratados con las Pistas Planas mejoraron hacia patrones Mesofaciales.

Los pacientes tratados con el Bionator, mejoraron su biotipo facial de Dolicofacial a Dólico suave y a Mesofacial. Ambos aparatos mostraron diferencias estadísticamente no significativas con la terapia funcional tras un año de aplicación. 
Tabla 7. Modificaciones en la biotipología facial producidos por el Bionator y las Pistas Planas.

\begin{tabular}{|c|c|c|c|c|c|c|c|c|c|c|}
\hline \multirow{3}{*}{ Biotipología facial } & \multicolumn{5}{|c|}{ Pistas Planas } & \multicolumn{5}{|c|}{ Bionator } \\
\hline & \multicolumn{2}{|c|}{ Antes } & \multicolumn{2}{|c|}{ Después } & \multirow{2}{*}{$\begin{array}{l}\text { Significación } \\
\text { estadística }\end{array}$} & \multicolumn{2}{|c|}{ Antes } & \multicolumn{2}{|c|}{ Después } & \multirow{2}{*}{$\begin{array}{l}\text { Significación } \\
\text { estadística }\end{array}$} \\
\hline & No & $\%$ & No & $\%$ & & No & $\%$ & No & $\%$ & \\
\hline Dólicofacial & - & - & - & - & - & 1 & 10 & - & - & 0,343 \\
\hline Dólico suave & 4 & 40 & 2 & 20 & 0,168 & 6 & 60 & 4 & 40 & 0,343 \\
\hline Mesofacial & 6 & 60 & 8 & 80 & 0,168 & 4 & 40 & 6 & 60 & 0,168 \\
\hline Total & 10 & 100 & 10 & 100 & - & 10 & 100 & 10 & 100 & - \\
\hline
\end{tabular}

Fuente: elaboración propia

\section{Discusión}

Al analizar los resultados del tratamiento de las maloclusiones de clase II división 1, después de utilizar el Bionator y las Pistas planas, se pudo constatar por los datos que aportan los ángulos estudiados del cefalograma de Steiner que la relación máxilo mandibular mejora tras la corrección funcional de la maloclusión. Lo anterior a causa fundamentalmente del SNB que continúa en clase II, valor angular que relaciona la mandíbula con respecto a la base del cráneo como estructura estática en el plano anteroposterior. Como se trata de pacientes en crecimiento, que se encuentran en periodo de dentición mixta, el proceso del desarrollo influye tanto en la etiopatogenia como en la corrección. Todo lo que contribuye a adelantar la mandíbula será favorable y, en sentido contrario, si la expresión del crecimiento tiende a la retrusión de la mandíbula, se empeora la clase II o dificulta la corrección. En términos cefalométricos, la rotación anterior de la mandíbula es positiva y la posterior negativa y opuesta al intento terapéutico. Estos resultados inciden positivamente pues sigue en la norma la relación máxilo mandibular, la cual muestra valores estadísticamente significativos en ambas terapéuticas. Estos resultados se ajustan al principio morfológico de las maloclusiones de clase II, y el intento terapéutico se centra en promover preferiblemente la mesialización de la arcada inferior para que alcance a la superior, y no viceversa, por lo tanto, cabe esperar el aumento del SNB y la poca variación del SNA en terapias funcionales de este tipo. La modificación tan favorable en el aumento del ángulo SNB y la poca variación del ángulo SNA coincide con investigaciones realizadas por autores como Borbón, et al. (21), así como Pakshir, et al. (18), Franchi (22), Han (23), Mew (24)' Malta et al. (25) y De la Rosa (26) cuando utilizaron el Bionator, de igual forma se obtuvieron resultados similares con las pistas planas por Alvarado Torres, et al. (27), así de forma similar con el aparato de Klammt en la investigación de Hernández Silva (28) y Araiza Contreras (29) con el aparato SN6. 
La modificación del ángulo ANB es significativo en ambos aparatos, lo que se justifica por el aumento del ángulo SNB a pesar de que continúa en clase II, esto debido a la reposición de la mandíbula, donde se muestran valores estadísticamente significativos al año de tratamiento, similares resultados obtuvieron Barnabé Raveli et al. (29) así como Han (23), Mew (24) y Malta, et al. (25) con la terapia del Bionator.

En este estudio predominó la tendencia al crecimiento horizontal antes y después de aplicar la terapéutica funcional con ambos aparatos, con diferencias estadísticamente significativas para la terapia con Pistas Planas. Clínicamente esta tendencia es favorable para el tratamiento ortopédico teniendo en cuenta que son pacientes portadores de maloclusiones de clase II, división I, que por lo general se caracterizan por el aumento del resalte y la sobremordida vertical; perfil convexo, retrognatismo mandibular con tendencia al crecimiento vertical. Consideramos que como la tendencia de crecimiento tiene su origen genético, y al realizar el tratamiento ortodóncico no se modifica ese patrón, sino que se reorienta el vector de crecimiento basándose en el diagnóstico del patrón esqueletal y la biotipología facial de los pacientes, estimulándole o bien frenándole el crecimiento a favor de los resultados esperados.

Esta mejoría de los valores cefalométricos logrados con las Pistas Planas más que con el Bionator puede atribuirse a la incomodidad de la rigidez del Bionator, bajo nivel de tolerancia, el grado de cooperación individual y de sus familiares, y luego porque es mejor la adaptación de los pacientes a las Pistas Planas ya que tienen como característica buscar un plano oclusal más paralelo provocando una desoclusión posterior, mejorando la oclusión y liberando los movimientos de lateralidad y de protrusión, logrando así el avance mandibular que favorece al adecuado desarrollo de los maxilares.

Al realizar las variaciones del posicionamiento del punto A en sentido anteroposterior respecto a la perpendicular del Nasion, se observó en este estudio una aparente retrusión de grado ligero con la terapéutica funcional en sentido anteroposterior con las Pistas Planas más que con el Bionator, obteniéndose significación estadística con el primer aparato y no con el segundo, ambos siguen quedando en la norma. Esto se explica por la posición más adelantada de la mandíbula lograda con estos tipos de aparatos, por lo que no hubo en realidad influencia sobre el punto A. Estudios realizados Barnabé Raveli, et al. (30) y Pakshir, et al. (18) donde utilizaron el Bionator, hallaron resultados similares al obtenido en la presente investigación, de igual forma Cruz Rivas (31) alcanzó valores análogos con las Pistas Planas; y Hernández Silva (28) con el aparato de Klammt.

Al año de tratamiento ortopédico se observó un aumento en la longitud efectiva de la mandíbula aún con hipoplasia utilizando ambas técnicas, pero particularmente la terapia con las Pistas Planas, con diferencias estadísticamente significativas 
corroborando la posición adelantada lograda del Pogonion en el plano sagital. Este aumento se debe a que una de las características de la maloclusión de clase II, es la oclusión posterior trabada y la ventaja que tienen estos aparatos es liberar ese obstáculo. Al lograr la desoclusion posterior, la mandíbula pudo, con su tendencia de crecimiento horizontal, expresarse ampliamente. Diferentes autores han logrado con el uso del Bionator un incremento de la longitud efectiva $(18,21-25,32,33)$. Así mismo con la utilización de las Pistas Planas se han hallado resultados favorables como los obtenidos por Cruz Rivas (31) y Alvarado Torres, et al. (27)

En este estudio, la altura facial anteroinferior disminuyó más en los pacientes tratados con las Pistas Planas en comparación con los tratados con el Bionator, con valores de significación estadística para el primer aparato y estadísticamente no significativa para el segundo. Tiene correlación inversa por el hecho de aumentar más la longitud efectiva de la mandíbula en la terapia funcional con las Pistas Planas. Esto se debe a las ventajas que tienen las Pistas Planas sobre el Bionator en realizar los movimientos mandibulares de avance favoreciendo su rotación antihoraria. En la presente investigación se coincide con estudios realizados por Alvarado Torres (27) donde utilizó Pistas Planas, y De La Rosa (26) y Barnabé Raveli, et al. (30) con el Bionator.

Al analizar los resultados de la convexidad facial al año de tratamiento según criterios de Ricketts se observó que ésta disminuyó con ambas terapias funcionales, siendo mayor la disminución con las Pistas Planas. Ambos resultados se encuentran en la norma, mostrando valores con significación estadística para las Pistas Planas y no así para el otro aparato, pero que mejoraron las medidas del patrón esqueletal de los pacientes objeto de estudio. Coincidimos con los estudios de Borbón Esquer, et al. (21), y De La Rosa (26) donde se aplicó terapia con Bionator, así como las investigaciones realizadas por Cruz Rivas (31) y Alvarado Torres (27) donde usaron las Pistas Planas, en el estudio de Alvarez et al. (5) con la utilización del Klammt.

Sin embargo, al comparar los valores obtenidos en la convexidad facial según Ricketts con los obtenidos por Conde Suárez et al. (34), en un estudio en oclusiones normales en niños de 9 años, en la provincia de Matanzas, se ve cómo los valores se acercan a la media obtenida en esa investigación.

La reducción de la convexidad facial en este estudio se debió fundamentalmente al avance del Pogonion como resultado del tratamiento ortopédico de las Pistas Planas, debido al mayor avance mandibular logrado con dichos aparatos, corroborando el aumento del ángulo cefalométrico de SNB.

Al realizar un análisis del ángulo del Eje Facial se observó el aumento de éste, sufriendo modificaciones estadísticamente significativas con la terapéutica funcional de las Pistas Planas que al concluir el tratamiento ortopédico se encuentra en la norma 
y mejoró. Con el uso del Bionator hubo cambios estadísticamente no significativos y una ligera apertura del eje, que constituyen una indicación de que está más influenciado su patrón de crecimiento genético que por los efectos ortopédicos del aparato ya que continúo en la norma. Estos valores en el cierre del eje facial son más evidentes en pacientes con patrones mesofaciales que en los dólicofaciales y es atribuido a la tendencia del crecimiento horizontal, influyendo en la mayoría de los pacientes objeto de estudio.

Estudios han demostrado que la poca variación en el ángulo del Eje Facial nos induce a pensar que la mandíbula crece pero de acuerdo con su patrón de crecimiento, de modo que, los mejores resultados se obtendrán en pacientes meso y braquifaciales (35).

Pakshir, et al. (18) reportaron que el ángulo del Eje Facial manifestó un valor de $88^{\circ}$, resultados inferiores alcanzados en este estudio en los pacientes que utilizaron el Bionator, sin embargo, superiores a los obtenidos por Borbón Esquer (21) que alcanzó un valor de $84^{\circ}$, Cruz Rivas (31) notó una apertura del eje facial, que difiere de la medida final de este estudio.

Cuando se evaluó el comportamiento de la profundidad facial tras un año de tratamiento, se observó que con la terapéutica funcional este ángulo aumentó con las Pistas Planas y el Bionator, mostrando valores estadísticamente significativos en ambas terapia. Este aumento se debe a la mejoría en el plano sagital del Pogonion que clínicamente se encontró más adelantada en los pacientes después de ser tratados ortopédicamente de su maloclusión de clase II, división I. Al comparar estos resultados con otros estudios vemos que coincidimos con De La Rosa (26). Borbón Esquer, et al. (215), Álvarez, et al. (5).

El ángulo de la atura facial Inferior disminuyó mostrando valores con significación estadística para la terapia con las Pista Planas como efecto del tratamiento, no hubo valores estadísticamente significativos en los pacientes tratados con el Bionator. Estos resultados corroboraron la tendencia al crecimiento horizontal, el cierre del eje facial, el aumento del ángulo SNB y la posición adelantada del Pogonion en el plano sagital encontrados en los pacientes objeto de estudio.

Respecto al ángulo de la altura facial inferior fueron reportados resultados similares a los hallados en esta investigación por Curbeira Hernández (36) con bloques gemelos y AAE de Klammt donde el ángulo está disminuido, al igual que De La Rosa (26) con Bionator. Sin embargo, no coincidimos con los valores alcanzados por Alvarado Torres, et al. (27) donde se elevan sus valores hasta $50^{\circ}$ al utilizar las pistas planas.

En este estudio, el ángulo nasolabial aumentó con ambas terapias sin mostrar significación estadística alguna. Este aumento se debe al componente labial del ángulo que varió su posición con el tratamiento por el cambio ortopédico y ortodóncico logrado 
con la terapia funcional de los aparatos al disminuir la proinclinación de los dientes anterosuperiores. Estos resultados coinciden con los alcanzados por Alvarez, et al. (5).

En la mayoría de los niños estudiados se presentó la tendencia de crecimiento horizontal y biotipo mesofacial, retrognatismo basal inferior con una desproporción entre las bases apicales. Analizando los valores cefalométricos esqueletales y de los tejidos blandos, se obtuvieron resultados más favorables en los pacientes que usaron las Pistas Planas en el tratamiento de las maloclusiones de clase II división I.

\section{Conclusiones}

Se pudo comprobar desde el punto de vista estadístico tras un año de tratamiento con la terapia funcional, que los pacientes a los que se les colocaron las pistas planas presentaron cambios craneofaciales estadísticamente más significativos en la mayoría de las medidas cefalométricas. Las mismas fueron: Steiner (SNB, ANB), Mc Namara (Punto A - Na Perpendicular, Pogonion - Na Perpendicular, Altura facial Anteroinferior), Ricketts (convexidad, eje facial, profundidad facial, altura facial inferior), en cuanto a los cambios en los tejidos blandos no se encontraron diferencias significativas para ninguno de las dos terapéuticas empleadas y el biotipo fue modificado positivamente con ambas aparatologías.

\section{Referencias}

1. Canut JA. Ortodoncia Clínica y Terapéutica. Barcelona: Masson; 2000. 389-424 p.

2. Morán V, Zamora O. Tipos de Maloclusiones y hábitos orales más frecuentes, en pacientes infantiles en edades comprendidas entre 6 y 7 años, de la E.B.N Los Salias, ubicada en San Antonio de los Altos, Edo. Miranda, Venezuela. Rev Latinoamericana de Ortodoncia y Odontopediatría. [Internet]. 2013 [consultado 10 de diciembre de 2017]. Recuperado en: http://www.ortodoncia.ws/publicaciones/2013/art4.asp

3. Arevalo JP, Sigüencia V, Bravo ME. Maloclusión Clase II-1, tratamiento ortodóncico. Revisión de la literatura. Rev Latinoamericana de Ortodoncia y Odontopediatría. [Internet]. 2014 [consultado 10 de diciembre de 2017]. Recuperado en: https://www.ortodoncia.ws/publicaciones/2014/art26.asp

4. Proffit WR. Malocclusion and dentofacial deformity in contemporary society. En: Proffit WR. Contemporary Orthodontics. St. Louis: Mosby, Elsevier; 2007. 3-22 p. 
5. Alvarez Mora I, Mora Pérez C, Morera Pérez A, Pentón García V, Blanco Hernández A, Villa Fernández M. Resultados del tratamiento de maloclusión clase II división 1 con activador abierto elástico de Klammt. Medisur. [Internet]. [consultado 10 de diciembre de 2017]; 15(3). Recuperado en: http://www.medisur.sld.cu/index.php/medisur/article/view/3322

6. Rodríguez C, Padilla MR. Manejo temprano de la maloclusión clase II división 2. Revisión de la literatura. Rev Estomatol. [Internet]. 2015 [consultado: 14 de abril de 2017]; 23(2):57-63. Recuperado en: http://bibliotecadigital.univalle.edu.co/bitstream/10893/9143/1/Manejo \%20temprano.pdf

7. Rothstein T, Yoon Tarlie C. Dental and facial skeletal characteristics and growth of males and females with Class II, Division 1 malocclusion between the ages of 10 and 14. Part I: Characteristics of size, form, and position. Orthod Dentofacial Orthop. 2001; 120(5): 542.

8. Graber TM, Melvin M, Sten Linder A, Donald E. Conceptos científicos y validación de los aparatos funcionales. En: Graber TM, Rakosi T, Petrovic AG. Ortopedia dentofacial con aparatos funcionales. Madrid: Harcourt Brace; 1998. 3-12 p.

9. Cuéllar Tamargo Y, Cruz Rivas Y, Llanes Rodríguez M, Suárez Bosch F, Perdomo Rodríguez M. Modificaciones labiales en pacientes Clase II División 1 tratados con Modelador Elástico de Bimler. Rev Haban Cienc Méd. [Internet]. 2015 [consultado: 14 de abril de 2017]; 14(1): 33-42. Recuperado en: http://scielo.sld.cu/scielo. php?script=sci_arttext\&pid=S1729-519X2015000100006\&lng=es.

10. Cuéllar Tamargo Y, Cruz Rivas Y, Llanes Rodríguez M, Suárez Bosch F, Santos Hernández O. Modificaciones del perfil facial en pacientes Clase ॥ División 1 tratados con Modelador Elástico de Bimler. Rev Haban Cienc Méd. [Internet]. 2014 [consultado: 14 de abril de 2017]; 13(6):845-54. Recuperado en: http://scielo.sld.cu/scielo. php?script=sci_arttext\&pid=S1729-519X2014000600005\&lng=es.5//

11. Cueto Salas A, Fernández Ysla R. Efectividad del Equiplán en el tratamiento del Síndrome de Clase II División 1. Rev Haban Cienc Méd. [Internet]. 2014 [consultado: 14 de abril de 2017]; 13(5):742-50. Recuperado en: http://scielo.sld.cu/scielo. php?script=sci_arttext\&pid=S1729-519X2014000500011\&lng=es

12. Mora Pérez C, Cruz Caballero R, Martínez Santos S, Rivas Pérez G. Maduración ósea en pacientes con maloclusión Clase II división 1 de Angle a partir del desarrollo dental. Medisur. [Internet]. 2012 [consultado: 9 de diciembre de 2017]; 10(4): 279-85. Recuperado en: http:// scielo.sld.cu/scielo.php?script=sci_arttext\&pid=S1727-897X2012000400002\&lng=es 
13. Jacobson A, Jacobson R. Twenty Centuries of cephalometry. Radiographic Cephalometry; From Basics to 3-D Imaging. Chicago: Quintessence; 2006. 13-32 p.

14. Faltin Jr K, Faltin RM, Baccetti T, Franchi L, Ghiozzi B, Mc Namara Jr JA. Long-term effectiveness and treatment timing for Bionator therapy. The Angle Orthodontist. 2003; 73(3):221-230. doi: 10.1043/0003-3219(2003)073<0221:LEATTF>2.0.CO;2

15. Otaño Lugo R. Aparatos Funcionales. En: Otaño Lugo R. Manual Clínico de Ortodoncia. La Habana: Ciencias Médicas; 2008. 155-184 p.

16. Graber T, Vanarsdall R, Vig K. Ortodoncia: Principios y Técnicas actuales. Mosby. 4ta. Edición. Madrid: Elsevier; 2006. 493-542,501-507 p.

17. Grohmann U. Bionator según Balters. En: Grohmann U. Aparatología en Ortopedia Funcional. Atlas Gráfico. Caracas: Amolca; 2002. 17-29 p.

18. Pakshir H, Mokhtar A, Darnahal A, Kamali Z, Behesti MH, Jamilian A. Effect of Bionator and Farmand Appliance on the Treatment of Mandibular Deficiency in Prepubertal Stage. Turkish J Orthod. 2017; 30:15-20. doi: 10.5152/TurkJOrthod.2017.1604.

19. Simoes AW. Ortopedia funcional de los maxilares. Sao Paulo: Artes Médicas Latinoamericana; 2004. 292-313 p.

20. Gregoret J, Tuber E. Instrumentación de la cefalometría estática lateral. En: Ortodoncia y Cirugía Ortognática: diagnóstico y planificación. Barcelona: Expaxs; 1997. 227-51 p.

21. Borbón Esquer CL, Gutiérrez Villaseñor J, Díaz Peña R. Empleo del aparato funcional Bionator para tratamiento de clase II esqueletal. Rev Tamé. [Internet]. 2013 [citado 14 de noviembre de 2017]; 2(5):159-164. Recuperado en: http://www.uan.edu.mx/d/a/publicaciones/revista_ tame/numero_5/Tam135-07.pdf

22. Franchi L, Pavoni C, Faltin K Jr, McNamara JA Jr, Cozza P. Long-term skeletal and dental effects and treatment timing for functional appliances in Class II malocclusion. Angle Orthod. [Internet]. 2013 [citado 24 de noviembre de 2017]; 83(2):334-340. doi: 10.2319/052912-450.1. Epub 2012 Aug 29

23. Han S, Choi YJ, Chung CJ, Kim JY, Kim KH. Long-term pharyngeal airway changes after Bionator treatment in adolescents with skeletal Class II malocclusions. Korean J Orthod. [Internet]. 2014 [citado 4 de noviembre de]; 44(1):13-19. doi: 10.4041/kjod.2014.44.1.13 
24. Mew J. Long-term skeletal and dental effects and treatment timing for functional appliances in Class II malocclusion. The Angle Orthodontist. [Internet]. 2013 [consultado 14 de abril de 2017]; 83(5): 334-340. doi: 10.2319/031609-156.1

25. Malta L.A, Baccetti T, Franchi L, Faltin K. McNamara JA. Long-Term Dentoskeletal Effects and Facial Profile Changes Induced by Bionator Therapy Angle Orthodontist. [Internet]. 2010 [consultado 9 de diciembre de 2017]; 80(1). Recuperado en: doi:10.2139/031609-156.1

26. De La Rosa Cabrera Y, Pérez García M. Efecto ortopédico del Bionator de california en pacientes con Clase II División. [Internet]. [consultado 8 de diciembre de 2017]. Recuperado en: http:// www.estomatologia2015.sld.cu/index.php/estomatologia/nov2015/paper/view/386/195

27. Alvarado Torres E, Gutiérrez Rojo JF, Rojas García AR. Uso de pistas planas en el tratamiento de una maloclusión de clase II con asimetría mandibular. Revista Tamé. [Internet]. 2017 [consultado 14 de noviembre de 2017]; 5(15): 529-531. Recuperado en: http://www.uan.edu. mx/d/a/publicaciones/revista_tame/numero_15/Tam1715-06c.pdf

28. Hernández Silva JA, Rodríguez Manjarrés C, Molina Betancourt N. Cambios cefalométricos obtenidos con activador abierto elástico de Klammt como tratamiento temprano de maloclusión clase II. Rev Nac Odontol. [Internet]. 2017 [consultado 4 de noviembre de 2017]; 13(25). doi: https://doi.org/10.16925/od.v13i25.1704

29. Araiza Contreras DJ, Mondragón Báez TD. Tratamiento ortopédico SN6 en adolescente con maloclusión clase II. Reporte de caso. Revista ADM. [Internet]. 2017 [consultado 10 de noviembre de 2017]; 74(3):152-158. Recuperado en: http://www.medigraphic.com/pdfs/adm/ od-2017/od173h.pdf

30. Barnabé Raveli, D, Schwartz, JP, Boamorte Raveli, T, dos Santos Pinto, A, Tompson, B. Skeletal and dental changes induced by bionator in early treatment of class II. Acta Scientiarum. Health Sciences. [Internet]. 2016 [consultado 24 de noviembre de 2017]; 38(2): 205-210. doi: 10.4025/actascihealthsci.v38i2.28763

31. CruzRivas Y, Gardón Delgado L, Marin Manso GM, Llanes Rodríguez M, Suárez Bosch F. Cambios cefalométricos en pacientes con clase II división 1 tratados con pistas planas. Rev. Habanera Cienc. Méd. [Internet]. 2006 [consultado 14 de noviembre de 2017]; 5(3). Recuperado en: http://www.ucmh.sld.cu/rhab/rhcmv5n3.htm 
32. Bigliazzi R, Franchi L, De Magalhães BertozAP, McNamara JA, Faltin K, Bertoz FA. Morphometric analysis of long-term dentoskeletal effects induced by treatment with Balters bionator. The Angle Orthodontist. [Internet]. 2015 [consultado 9 de noviembre de 2017]; 85(5):790-798. doi: https://doi.org/10.2319/080714-554.1

33. Gorucu Coskuner H., Ciger S. Three-dimensional assessment of the temporomandibular joint and mandibular dimensions after early correction of the maxillary arch form in patients with Class II division 1 or division 2 malocclusion. Rev Korean J Orthod. [Internet]. 2015 [consultado 10 de diciembre de 2017] 45(3):121-129. doi: 10.4041/kjod.2015.45.3.121

34. Conde Suárez HF, Valentín González F, Sánchez Gay JM. Normas del cefalograma de Ricketts en niños de Matanzas. Rev Cubana Estomatol. [Internet]. 2013 [consultado 10 de diciembre de 2017]; 50(4). Recuperado en: http://www.revestomatologia.sld.cu/index.php/est/rt/ printerFriendly/149/44

35. La LuceM. El significado clínico del análisiscefalométrico. En:La LuceM. Terapias Ortodónticas. Torinese: AMOLCA; 2002. 36-46 p.

36. Curbeira Hernández EM, Mora Pérez C. Activador abierto elástico de Klammt y gemelos en el tratamiento de la maloclusión de clase II. Medisur. [Internet]. 2009; 7(1). Recuperado en: http://www.medisur.sld.cu/index.php/medisur/article/view/487 